\title{
Investigation of time dependence of dissipation and strain induced crystallization in natural rubber under cyclic and impact loading
}

\author{
K. Schneider, L. Zybell, J. Domurath \& G. Heinrich \\ Leibniz-Institut fuer Polymerforschung Dresden e.V., Dresden, Germany
}

S. V. Roth, A. Rothkirch, W. Ohm

Photon Science at DESY, Hamburg, Germany

\begin{abstract}
Strain induced crystallization (SIC) is one of the special features of natural rubber (NR), responsible for its outstanding mechanical performance. Especially, for the short term behavior of NR, e.g. within a rolling tire, the kinetics of SIC plays an important role. SIC can be observed to some extend by thermography, but it is not directly possible to separate the heating effects accompanying SIC from dissipative heating of the rubber material (LeCam et al. 2015, Spratte et al., 2017). By means of synchrotron x-ray diffraction, SIC can be monitored during deformation including cyclic deformations and impact loading with a time resolution of less than 10 ms (Brüning at al., 2013). Combining this method with thermography enables the separation of processes of structure formation and dissipation. This can serve as a basis for a more realistic modelling of the dynamic and thermomechanical behavior of natural rubber.
\end{abstract}

Within the presentation we report about investigations of cyclic loadings of differently carbon black (CB) filled NR samples with respect to SIC, melting of the crystallites as well as the dissipative heating and convective cooling of the rubber materials.

\section{INTRODUCTION}

Strain induced crystallization (SIC) is one of the reasons for the outstanding properties of natural rubber. Due to this crystallization the stiffness of the material is increased mainly on the positions of highest strain leading to a self-reinforcing effect. But due to the kinetics of SIC this reinforcing effect has a certain time dependence.

SIC can be followed directly by wide angle x-ray scattering (WAXS). Because of the increase in brilliance of contemporary synchrotron radiation sources and the effort in detector sensitivity and read out time, nowadays the exposure time for a single pattern can be reduced to the millisecond regime.

Thermography enables a space-resolved measurement of surface temperatures. By suitable thermodynamic models it is possible to derive from those temperatures the thermal behavior of samples caused by thermal active processes like energy dissipation or crystallization/melting of crystallites in a good approximation. The high time resolution of contemporary infrared cameras enables to follow time dependent thermal processes in detail.
By the combination of deformation experiments with simultaneous synchrotron $\mathrm{x}$-ray scattering as well as thermography, it becomes now experimentally possible to follow SIC and simultaneously to separate the crystallization dependent heat production or adsorption from dissipative processes in the material and to follow the time dependence of these processes.

Due to the high time resolution as a consequence of the high brilliance of the synchrotron sources, meanwhile it becomes possible to investigate practically interesting loading regimes, like cyclic impact loading with frequencies of e.g. $10 \ldots 100 \mathrm{~Hz}$, which is representative for the dynamic loading of a rolling tire.

\section{EXPERIMENTAL}

\subsection{Materials}

Unfilled and carbon black (CB) filled natural rubber compounds were investigated. The filler content was 0, 20 and 50 (weight) parts CB per hundred rubber (phr), they are named NR, NR20 and NR50 respectively. 


\subsection{Sample geometry}

To guaranty a well-defined clamping, specimen with a cylindrical bulge were used. The parallel length of the specimen was $10 \mathrm{~mm}$, the thickness 1.5 $\mathrm{mm}$ and the width $2.0 \mathrm{~mm}$.

\subsection{Experimental arrangement}

The investigations were carried out at beamline P03, DESY, Hamburg (Schneider, G.J., 2010, Buffet, 2012). The general outline of the experiments is shown in Figure 1, it was described elsewhere (Schneider, K., 2010).

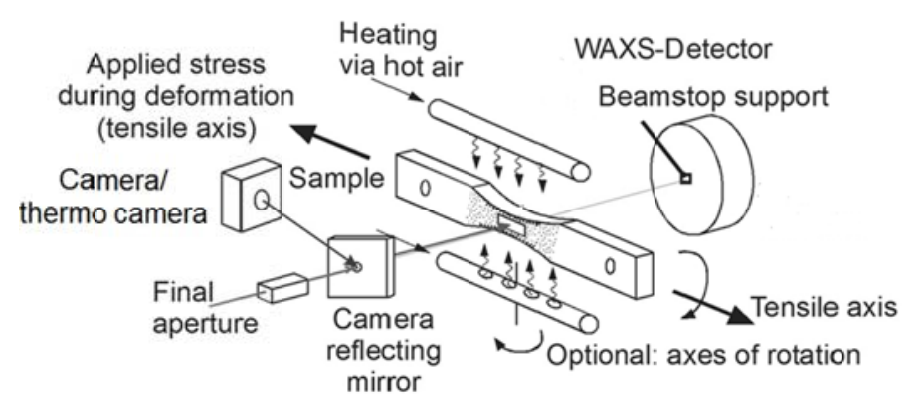

Figure 1: Experimental setup for the simultaneous estimation of SIC and temperature changes during deformation.

A home-made tensile machine enables quasistatic as well as dynamic loading of the specimen. Optionally the loading can be performed pneumatically driven impact-like.

\subsection{Estimation of relative crystallinity}

The WAXS pattern of NR is mainly characterized by an amorphous halo. During stretching a preorientation of the chains takes place which results in a change in the azimuthal intensity within the amorphous halo. Reaching the onset strain of SIC, the sharp reflexes of the crystallites appear mainly within the region of the amorphous halo. The intensity of the peaks refers to the degree of crystallinity. With ongoing stretching also the thickness of the sample reduces, roughly proportional to $\lambda^{-1 / 2}$, where $\lambda$ is the stretching ratio with $\lambda=1+\varepsilon$ with the strain $\varepsilon$. Because the scattering signal is proportional to the scattering volume, one has to account for decreasing sample thickness when comparing intensities of crystalline reflexes.

For a first qualitative discussion the intensity of the crystalline peaks was estimated as the cumulative intensity within the region of the crystalline reflexes subtracted by the intensity of an equivalent region of the pattern in direct vicinity to the relevant peaks at same scattering angle.

The relevant crystallite peaks of SIC and the regions, which were used for the estimation of relative crystallinity, are shown in Figure 2.

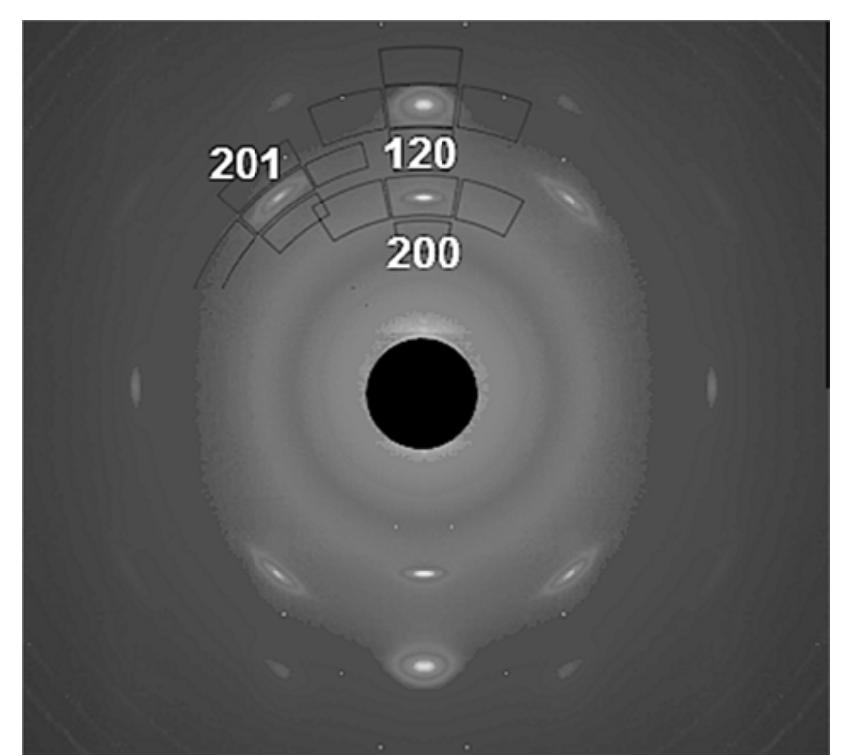

Figure 2: WAXS pattern of NR with the reflexes of SIC (stretching in horizontal direction) and their crystallographic indices as well as the regions which were used for crystallinity estimation.

\section{RESULTS}

\subsection{Free cooling behavior of the specimen}

After an impact loading or unloading of a sample, where spontaneous heating or cooling is induced, there follows a thermal equilibration to the surrounding. The heat exchange is mainly driven by the temperature difference between the surface and the surrounding, the surface area, heat transmission coefficient and the heat capacity of the sample. Under the preposition of thin samples with lateral dimensions which are large compared to the thickness, the time dependent temperature of the free cooling or heating sample can be approximated by

$$
T(t)=T_{\infty}+\left(T_{0}-T_{\infty}\right) \cdot \exp (-t / \tau),
$$

with the characteristic time constant

$$
\tau=\frac{m \cdot c}{\alpha \cdot A}=\frac{\rho \cdot c \cdot d_{0}}{\alpha \cdot \sqrt{\lambda}}=\frac{\tau_{0}}{\sqrt{\lambda}},
$$

where $\mathrm{m}$ is the mass of the sample, $c$ the specific heat capacity, $A$ the actual surface transferring the heat, $\alpha$ is the heat transmission coefficient, $\rho$ the density of the material, $\lambda$ the stretch ratio and $d_{0}$ the initial thickness of the sample. $T_{0}$ and $T_{\infty}$ are the initial and the equilibrium (ambient) temperature.

For a NR sample with an initial thickness of 1.5 $\mathrm{mm}$ it follows $\tau_{0}=13.6 \mathrm{~s}$. 
If the impact load crosses the threshold strain at which SIC starts, the time dependent heat production caused by the SIC overlays the heating of the sample caused by entropy elasticity and dissipation. Therefore the tip in the temperature-time curve is not so sharp like in the case of unloading, where the crystallites were dissolved immediately.

\subsection{Time dependence of SIC}

The typical time dependence of SIC in NR20 after impact loading is shown in Figure 3.

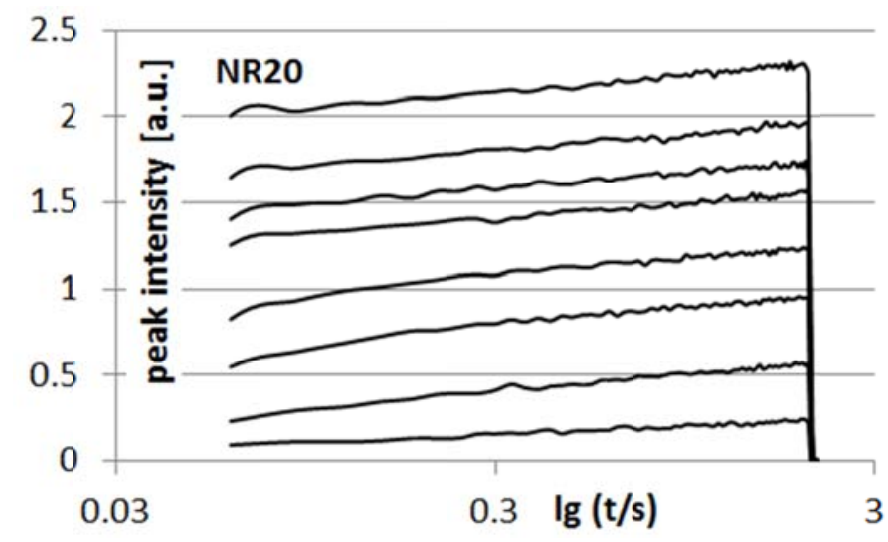

Figure 3: Time dependent increase of the intensity of the $\left(\begin{array}{lll}1 & 2 & 0\end{array}\right)$-peak which is proportional to the strain induced crystallinity after impact loading of NR 20 for $2 \mathrm{~s}$, stretching ratios are (from bottom curve up) 3.5, 4.5, 5.0, 5.5, 6.0, 6.5, 7.0 and 7.5

The kinetics of strain induced crystallization can be described by

$$
\frac{d \Phi}{d t}=\left(k \cdot\left(\Phi_{f}-\Phi\right)\right)^{n},
$$

with the crystallinity $\Phi$, the equilibrium crystallinity $\Phi_{\mathrm{f}}$, a constant $k$ and the exponent $n$. The dissolution of the crystallites appears suddenly during unloading below a certain strain threshold; see also Brüning 2015. In the present case the exponent $\mathrm{n}$ for $\lambda>4$ is about 0.2 .

\subsection{SIC and temperature evolution during cyclic loading}

Below the onset of SIC there is a certain amount of energy dissipated within each loading cycle, where the energy loss within the mechanical cycle is identical to the heat input into the specimen. After a certain number of cycles, a final equilibrium temperature is reached where the heat loss to the surrounding is equal to the dissipated mechanical energy.

For triangular loading-unloading cycles with stepwise increased strain, the evolution of stress, SIC and temperature as a function of stretching ratio are shown in Figures 4 to 6. For each strain level the loading-unloading cycles are repeated 3 times.

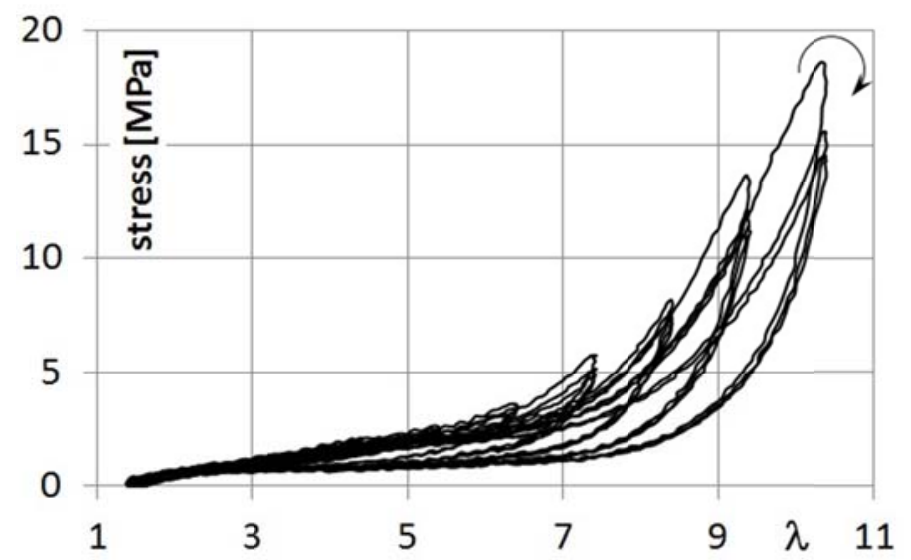

Figure 4: Stress vs. stretching ratio for NR during repeated cyclic loading with triangular strain profile to $\lambda=4.4,5.4,6.4,7.4,8.4,9.4$ and 10.4 .

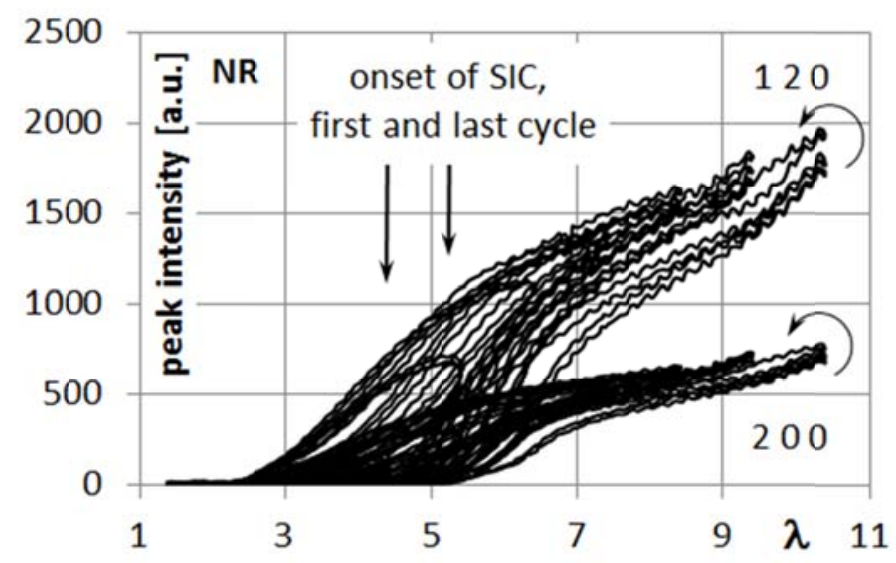

Figure 5: Peak intensity which is proportional to the strain induced crystallinity during cyclic loading of NR with triangular strain profile, evaluation of the two indicated different crystalline reflexes.

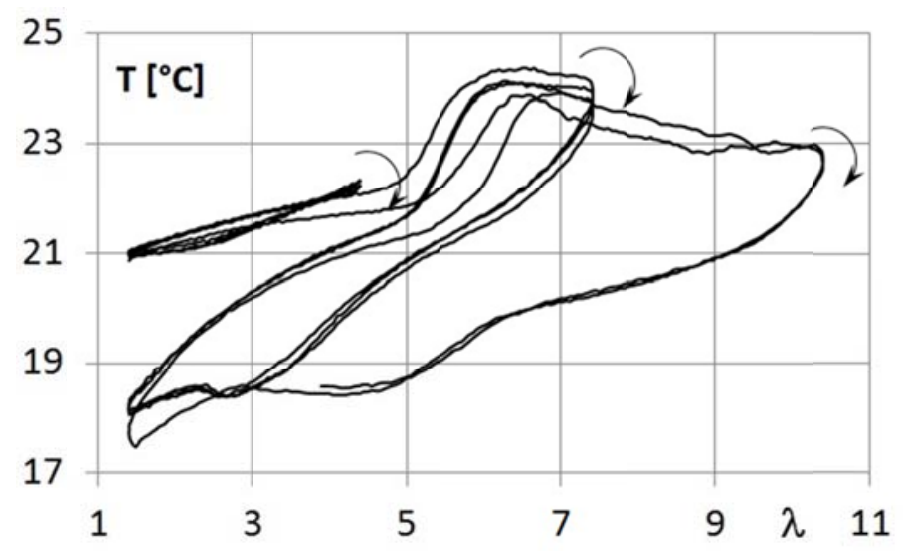

Figure 6: Cyclic loading of NR with triangular strain profile, temperature evaluation in dependence of the stretching ratio, $\lambda=4.4,7.4$ and 10.4

The stress-stretch curves show the commonly known behavior with hysteresis. The first loading 
cycle to a new strain level is always slightly higher than the following cycles.

The curves of peak intensity vs. stretch, which have the same form like the crystallinity-stretch curves show the onset of SIC at a stretch of about 3 to 4 and a hysteresis during unloading, see Fig. 5. After some cycles to higher strains the onset value for SIC shifts to higher values.

Up to now it was not reported, that the crystallization process shows a different behavior at lower and higher strains. After an initial nearly linear increase of crystallinity with stretch the crystallization process seems to reaches a saturation. That means the crystallinity remains nearly constant during further stretching or increases only much slower than before. During unloading, nearly no hysteresis is observable in this range.

The temperature-stretch curves show a behavior which correlates strongly with the crystallizationstretch curves.

In the range before the onset of SIC, only a very small heating of the sample can be observed during a loading-unloading cycle. The final temperature is slightly higher than the initial one, the whole cycle is superposed by a steady heat release to the surrounding.

During the classical SIC, that is the first region of the crystallization curve, see Fig. 6, the system releases the heat of crystallization resulting in a strong increase of temperature associated with increased heat flux to the surrounding. There the crystallinity $\Phi$ is coupled with the specific crystallization heat $\Delta h_{c r}$ and the increase of temperature $\Delta T$ by

$$
\Delta h_{c r} \cdot \Phi=c_{p} \cdot \Delta T .
$$

Because $\Delta h_{c r}$ is not really known, the estimated crystallinity is shown here always only in arbitrary units.

Above a stretch of about 6 the temperature falls approximately exponential. This means that there is nearly no further heat production or at least a heat production which is much less than heat flux according to the natural cooling down.

\subsection{SIC and temperature evolution during cyclic impact loading}

The kinetics of SIC during cyclic impact loading is rarely independent on the holding times at low and high strain under the condition that the low strain stage is below the onset of SIC.

During stretching the thickness of the sample reduces and therefore also the absorption changes. This enables an accurate synchronization of the impact device with the scattering equipment and with the resulting vibrations of the sample. Interestingly, it seems here that the SIC requires a certain nucleation time, which impedes a strong reproducibility of the crystallinity vs. time curves.

So, at very short impacts of less than $10 \mathrm{~ms}$ the reproducibility of the crystallinity and also the reinforcement effect becomes less reproducible.

\subsection{Influence of filler comtent on SIC and temperature evolution during cyclic stretching}

The filler content has a characteristic impact on the SIC vs. strain curves. On the one hand, the total amount of crystallizable rubber becomes less, on the other hand, due to the distribution of the rubber between the filler, strain amplification takes place locally causing a shift of the onset of SIC to lower strains, see Fig. 7.
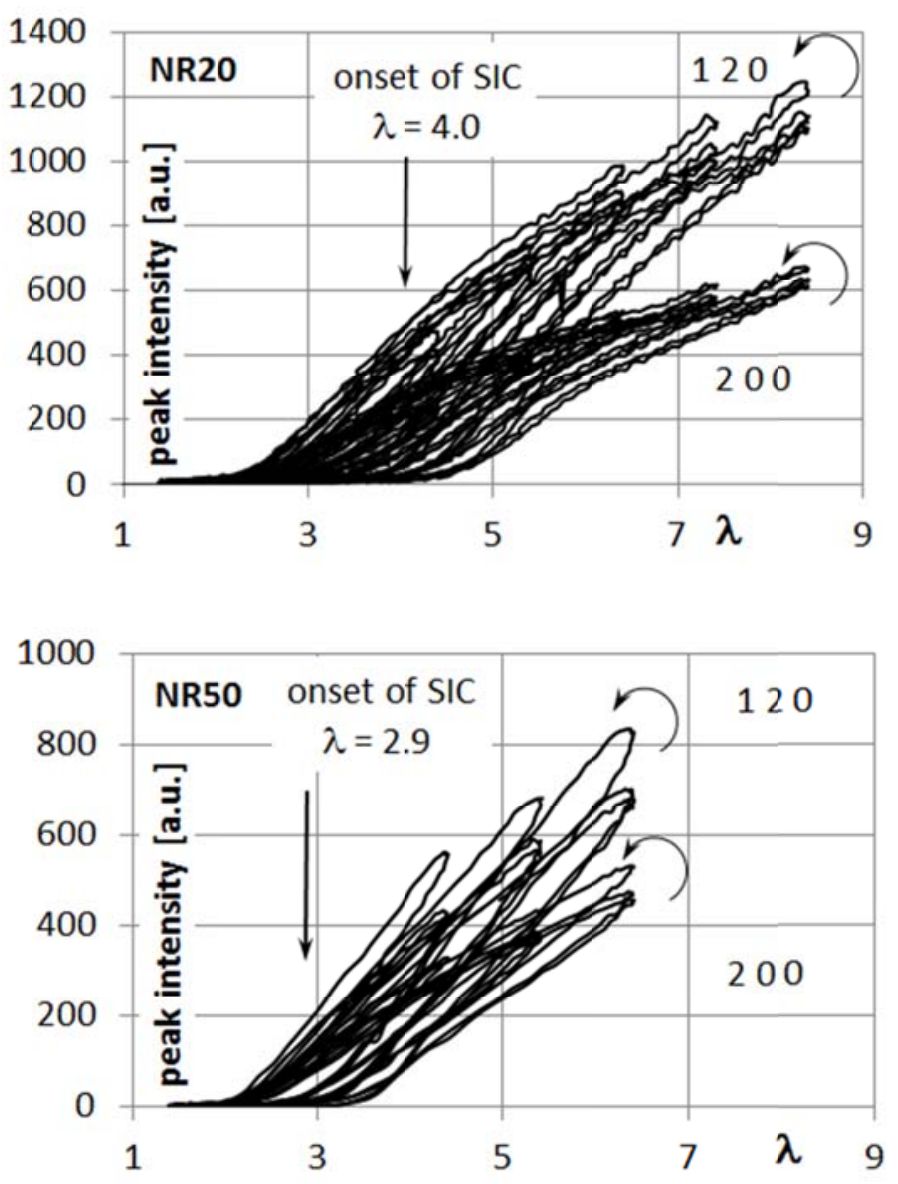

Figure 7: Peak intensity which is proportional to the strain induced crystallinity during cyclic loading of NR20 (top) and NR50 (bottom). The intensities of the (1 20 ) as well as the (2 00 ) reflexes are shown.

\section{DISCUSSION}

The stress-strain behavior of NR must be divided into 3 regions resp. regimes. In the first region, before the onset of SIC, the material behaves hyperelastically with superimposed dissipative behavior. This dissipative behavior is responsible for continu- 
ous heating of the samples during cyclic loading. According to the heat transfer to the surrounding, the sample finally reaches an asymptotic temperature by this process, normally above the ambient temperature.

Despite this dissipation the material behaves hyperelastically, the energy stored by entropy-elasticity is responsible for a reversible heating and cooling of the sample.

During SIC the heat of crystallization is responsible for a strong heating of the sample in the second region, during subsequent unloading the same amount of energy is released for the dissolution of the crystallites causing a cooling of the sample.

After reaching a certain degree of crystallinity, which is dependent on the individual rubber network and the filler content, in a third region a further deformation of the sample obviously behaves like an energy-elastic material without further heating of the sample.

In filled rubber materials the distinction of the stages of the strain crystallizing materials is less apparently like in the unfilled NR.

For highly dynamic loading of rubber the dynamics of SIC must be taken into account. Here, mainly the question of nucleation of SIC must be further investigated.

\section{CONCLUSIONS}

Coupling of thermography and synchrotron x-ray scattering during cyclic tensile tests is a powerful tool to separate dissipative processes within unfilled as well as filled rubber materials from structural changes like strain induced crystallization. For a quantitative evaluation of the energy balance, it is important to take into account the natural heat transfer to the surrounding. For a quantitative evaluation of the thermography measurements, not only the geometry change of the samples has to be taken into account, but also the changes in the emission coefficient of the samples due to deformation, in particular in the case of unfilled natural rubber.

The hysteresis of the stress-strain curves is similar to the hysteresis in the SIC. But at a certain strain the crystallization slows down or stops, while the sample temperature is mainly estimated by free cooling.

\section{ACKNOWLEDGEMENT}

DESY, Hamburg (Germany) is acknowledged for beamtime within the long term proposal II-2015 0042.

\section{REFERENCES}

Brüning, K., Schneider, K. \& Heinrich, G., In-situ Characterization of Rubber during Deformation and Fracture, chap. 2 in Grellmann, W. et al. (eds.) 2013. Fracture Mechanics and Statistical Mechanics of Reinforced Elastomeric Blends. LNACM 70, SpringerVerlag Berlin, Heidelberg

Brüning, K. Schneider, K., Roth, S., Heinrich, G. 2015. Kinetics of strain-induced crystallization in natural rubber: A diffusion-controlled rate law. Polymer 72: 52-58.

Buffet, A., Rothkirch, A., Döhrmann, R., Körstgens, V., Abul Kashem, M. M., Perlich, J., Herzog, G., Schwartzkopf, M., Gehrke, R., Müller-Buschbaum, P. \& Roth S. V. 2012. P03, the Microfocus and Nanofocus X-ray Scattering (MiNaXS) beamline of the PETRA III storage ring: The microfocus endstation, J. Synchr. Radiation 19: 647.

LeCam, J.B., Samanca Martinez, J.R., Balandraud, X., Toussaint, E. \& Caillard, J. 2015. Revisiting the mechanisms involved in rubber deformation using experimental thermomechanics. In B. Marvalová I. Petríková (eds), Constitutive Models for Rubbers IX: 3-11. Boca Raton et al.: CRC Press.

Schneider, G. J., Vollnhals, V., Brandt, K., Roth, S. V. \& Göritz, D. 2010. Correlation of mass fractal dimension and cluster size of silica in styrene butadiene rubber composites, J. Chem. Phys. 133: 094902.

Schneider, K. 2010. Investigation of Structural Changes in Semi-Crystalline Polymers During Deformation by Synchrotron X-Ray Scattering. J. Polymer Sci. B 48: 15741586

Spratte, T., Plagge, J., Wunde \& M., Klüppel, M. 2017. Investigation of strain-induced crystallization of carbon black and silica filled natural rubber composites based on mechanical and temperature measurements. Polymer 115: 12-20. DOI: 10.1016/j.polymer.2017.03.019 